\title{
The Association of Low Blood Glucose and Low Serum Cortisol Levels in Severely III Children Admitted to Tertiary Referral Hospitals in Malawi: A Case-Control Study
}

\author{
Fatsani Ngwalangwa,,${ }^{1,2^{*}}$ Clifford Katumbi, ${ }^{3}$ Queen Dube, ${ }^{4}$ Josephine Langton, ${ }^{1}$ Tim Baker, ${ }^{1,5,6}$ Annika Janson, ${ }^{7,8}$ and \\ Helena Hildenwall ${ }^{5,7,9}$
}

\begin{abstract}
${ }^{1}$ Department of Paediatrics, University of Malawi, College of Medicine, Blantyre, Malawi; ${ }^{2}$ Department of Public Health, University of Malawi, College of Medicine, Blantyre, Malawi; ${ }^{3}$ Clinical Department, Light House Trust, Kamuzu Central Hospital, Lilongwe, Malawi; ${ }^{4}$ Department of Paediatrics, Queen Elizabeth Central Hospital, Blantyre, Malawi; ${ }^{5}$ Karolinska Institutet, Department of Global Public Health, Stockholm, Sweden; ${ }^{6}$ Department of Clinical Research, London School of Hygiene and Tropical Medicine, London, United Kingdom; ' Astrid Lindgren Children's Hospital, Karolinska University Hospital, Stockholm, Sweden; ${ }^{8}$ Karolinska Institutet, Department of Women's and Children's Health, Stockholm, Sweden; ${ }^{9}$ Karolinska Institutet, Department of Clinical Science, Intervention and Technology, Huddinge, Sweden
\end{abstract}

\begin{abstract}
Low blood glucose concentrations $<5 \mathrm{mmol} / \mathrm{L}$ in severely ill children presenting to hospitals in low-income countries are associated with mortality. Adrenal insufficiency with low cortisol levels may contribute to low blood glucose concentrations. Understanding the association between low cortisol and low blood glucose may assist in improving guidelines for management of severely ill children. The study aimed to determine the association between low serum cortisol and low blood glucose in severely ill children. A matched case-control study of children aged 1 month to 15 years was conducted at two tertiary hospitals in Malawi. Cases were children with blood glucose $<5 \mathrm{mmol} / \mathrm{L}$. Two age-matched controls with blood glucose of $\geq 5-15 \mathrm{mmol} / \mathrm{L}$ were enrolled per case. Low cortisol was defined as serum cortisol of $<25 \mu \mathrm{g} / \mathrm{dL}(690$ $\mathrm{nmol} / \mathrm{L})$ and adrenal insufficiency as serum cortisol of $<10 \mu \mathrm{g} / \mathrm{dL}(276 \mathrm{nmol} / \mathrm{L})$. A total of 54 cases and 108 controls were enrolled with, median age of 2.8 years (interquartile range [IQR]: 1.7-4.4). The median cortisol level was $58.7 \mu \mathrm{g} / \mathrm{dL}$ (IQR: 42.3-61.8) in cases and $40.9 \mu \mathrm{g} / \mathrm{dL}$ (IQR: 33.7-51.2) in controls $(P=0.911)$. The proportion of low cortisol was $4 / 54$ $(7.4 \%)$ in cases and 9/108 (8.3\%) in controls. Logistic regression shows no association between low cortisol and low blood glucose (adjusted odds ratio: 0.33; 95\% confidence interval, 0.04-3.02). Results suggest that there is no association between low cortisol and low blood glucose among severely ill children presenting to hospitals in Malawi. The reason for low blood glucose needs further investigation.
\end{abstract}

\section{INTRODUCTION}

A normal blood glucose level ensures sufficient energy supply to vital organs in the body, especially the brain. ${ }^{1}$ The blood glucose level is maintained by the interplay of the glucoselowering action of insulin and the glucose-raising action of the counter-regulatory hormones cortisol, catecholamines, glucagon, and growth hormone. ${ }^{2}$ Low blood glucose may occur if there is an imbalance in the regulatory hormones or if there are diminished levels of glucose or its substrates in the body. ${ }^{2}$

Hypoglycemia is a common metabolic condition in pediatric emergencies in low-income countries. ${ }^{3}$ The World Health Organization $(\mathrm{WHO})$ currently defines pediatric hypoglycemia as a blood glucose value $<2.5 \mathrm{mmol} / \mathrm{L}$ or $<3 \mathrm{mmol} / \mathrm{L}$ in a severely malnourished child. ${ }^{4}$ The prevalence of hypoglycemia at admission among African pediatric patients has been reported as up to $7.3 \% .^{5}$ Hypoglycemia may result in seizures, altered consciousness, coma, ${ }^{3}$ as well as increased risk of mortality. ${ }^{5,6}$ Studies have shown $>3$-fold higher mortality also in children with "low glycemia," which is defined as a blood glucose above the WHO cut-off of $2.5 \mathrm{mmol} / \mathrm{L}$, with a variable upper limit of up to $5 \mathrm{mmol} / \mathrm{L}^{7-9}$ This has led to the questioning of the current cut-off for hypoglycemia. However, a recent study by our group did not show any mortality reductions from treating lowglycemic children with intravenous dextrose. ${ }^{10}$

A normal response to acute illness is an increased blood glucose due to the release of cortisol in response to the acute stressor caused by the illness. ${ }^{2}$ However, as the acute illness progresses, the adrenal glands may become unable to release

* Address correspondence to Fatsani Ngwalangwa, Department of Public Health, University of Malawi, College of Medicine, P/Bag 360, Blantyre, Malawi. E-mail: fatsa400@yahoo.com sufficient amounts of cortisol, manifesting in adrenal insufficiency and low levels of cortisol with possible consequences on glucose levels. Studies in adults have demonstrated a positive correlation between low serum cortisol, severity of illness, and an increased risk of death. ${ }^{11,12}$ Prevalence rates of adrenal insufficiency determined by low cortisol levels of up to $60 \%$ of critically ill adult patients with sepsis have been reported in another study. ${ }^{13}$ However, different cut-offs have been used to define adrenal insufficiency. ${ }^{14,15}$

In addition to substrate depletion, we hypothesized that low glucose levels in severe sickness were related to an exhausted stress response and low cortisol levels causing an inability to counter-regulate the low glucose and hypoglycemia. This study was conducted to determine the association between low blood glucose and low serum cortisol among severely ill children admitted to two tertiary referral hospitals in Malawi.

\section{METHODS}

This was a case-control study of severely ill children aged 1 month to 15 years presenting to the pediatric emergency departments at Queen Elizabeth Central Hospital (QECH) and Zomba Central Hospital $(\mathrm{ZCH})$ in Malawi from March 2019 to June 2020.

Queen Elizabeth Central Hospital is the largest referral hospital in Malawi, with 1,000 inpatient beds. The pediatric department serves 100,000 children a year for various illnesses, with approximately 24,000 admissions annually. All severely ill children with any WHO emergency $\operatorname{sign}^{16}$ are admitted via the resuscitation room of the pediatric accident and emergency unit and later transferred to the ward after stabilization. Zomba Central Hospital is a 500-bedded referral hospital, and pediatric patients are admitted from the under-five clinic or directly to the ward. Approximately 
16,000 children are admitted at Zomba Central Hospital every year. $^{17}$

The Malawi government rolled out WHO's Emergency Triage, Assessment and Treatment guidelines for the management of severely ill children in all government hospitals in 2009. ${ }^{18}$ According to the standard procedures in both hospitals, children who present with any $\mathrm{WHO}$ emergency $\operatorname{sign}^{16}$ (obstructed/absent breathing, central cyanosis, coma, convulsion, shock, severe dehydration) are identified by the hospital triage nurse upon arrival to the hospital for immediate assessment, treatment, and stabilization. After triaging, all children with severe illness have their vital signs assessed, and a test for capillary blood glucose at point of care is performed. Other emergency blood samples, like malaria Rapid Diagnosis Test and Packed Cell Volume, are taken if clinically indicated.

For this study, all children 1 month to 15 years with any WHO emergency $\operatorname{sign}^{16}$ and a valid blood glucose result were screened by a study nurse at the pediatric emergency department for possible recruitment as soon as they had been triaged. The participants were recruited within weekday working hours from 7:30 AM to 4:30 PM. Potential participants and their guardians were enrolled after having received information about the study and provided their consent.

Cases were children with at least one WHO emergency sign and a blood glucose level of $<5.0 \mathrm{mmol} / \mathrm{L}$ at presentation.
Each case was matched with two controls who also presented with at least one WHO emergency sign but had a blood glucose between 5.0 and $15.0 \mathrm{mmol} / \mathrm{L}$. A blood glucose of up to $15.0 \mathrm{mmol} / \mathrm{L}$ was considered nonpathological because the study population was severely ill, and hyperglycemia commonly occurs in severe illness. ${ }^{19,20}$ Matching was done for age, accepting \pm 6 months difference for controls of cases below 5 years of age and \pm 1 year for cases from 5 years and above. Children were not included if presenting with any of the following study exclusion criteria: 1) known diagnosis of diabetes or any other glucose metabolism disorder, 2) adrenal tumor, 3) thyroid disease, 4) insulin or any other diabetic medication, 5) steroid medication, or 6) severe acute malnutrition.

All enrolled children had information collected on age, sex, WHO emergency signs at presentation, vital signs (pulse rate, respiratory rate, oxygen saturation, body temperature, and Blantyre Coma Score), fasting hours (as reported by the guardian), and whether the participant was referred from another facility. Blantyre Coma Score is a pediatric coma scaling system that was initially developed for children with cerebral malaria but is now widely used in general pediatric care. ${ }^{21}$ Guardians were asked questions on the child's past medical history, previous admissions, and the presence of any chronic illness (e.g., cerebral palsy, cancer, sickle cell anemia, tuberculosis, HIV, heart disease, or congenital anomalies). A brief

TABLE 1

Characteristics of study participants

\begin{tabular}{|c|c|c|c|}
\hline Variable & All $(N=162)$ & Cases $(N=54)$ & Controls $(N=108)$ \\
\hline \multicolumn{4}{|l|}{ Sex, $n(\%)$} \\
\hline Female & $61(37.6)$ & $18(33.3)$ & $43(39.3)$ \\
\hline \multicolumn{4}{|l|}{ Age, $n(\%)$} \\
\hline 1 month -5 years & $129(79.6)$ & $43(79.6)$ & $86(79.6 .6)$ \\
\hline $5-15$ years & $33(20.4)$ & $11(20.4)$ & $22(20.3)$ \\
\hline Median age (IQR) & $2.8(1.7-4.4)$ & $2.8(1.7-4.4)$ & $2.8(1.6-4.3)$ \\
\hline \multicolumn{4}{|l|}{ Time of sample collection } \\
\hline 7:30 AM-12:00 PM & $82(50.6)$ & $29(53.7)$ & $53(49.1)$ \\
\hline$>12: 00$ PM-4:30 PM & $80(49.4)$ & $25(46.3)$ & $55(50.9)$ \\
\hline \multicolumn{4}{|c|}{ One or more severely deranged vital sign, ${ }^{\star} n(\%)$} \\
\hline Yes & $122(76.3)$ & $38(71.7)$ & $84(78.4)$ \\
\hline \multicolumn{4}{|l|}{ WHO emergency signs, $\uparrow n(\%)$} \\
\hline Obstructed breathing & $3(1.7)$ & $0(0.0)$ & $3(2.8)$ \\
\hline Central cyanosis & $12(7.4)$ & $1(1.9)$ & $11(10.3)$ \\
\hline Respiratory distress & 63 (38.9) & 18 (33.3) & $45(41.7)$ \\
\hline Coma & $73(45.1)$ & 28 (51.9) & $45(41.7)$ \\
\hline Convulsion & 69 (42.6) & 25 (46.3) & $44(40.7)$ \\
\hline Shock & $9(5.6)$ & $3(5.6)$ & $6(5.6)$ \\
\hline Severe dehydration & $11(6.8)$ & $3(5.6)$ & $8(7.4)$ \\
\hline \multicolumn{4}{|l|}{ Fasting time, $n(\%)$} \\
\hline$<8$ hours & $80(49.4)$ & $22(40.7)$ & $58(53.7)$ \\
\hline$\geq 8$ hours & $82(50.6)$ & $32(59.3)$ & $50(46.3)$ \\
\hline \multicolumn{4}{|l|}{ Chronic illnesses, $n(\%)$} \\
\hline No & $151(93.2)$ & 52 (96.3) & 99 (91.7) \\
\hline Yes & $11(6.8)$ & $2(3.7)$ & $9(8.3)$ \\
\hline \multicolumn{4}{|l|}{ Referred, $n(\%)$} \\
\hline No & 25 (15.4) & $9(16.7)$ & $16(14.8)$ \\
\hline Yes & $137(84.6)$ & 45 (83.3) & $92(85.2)$ \\
\hline \multicolumn{4}{|l|}{ Blood glucose level, $n(\%)$} \\
\hline$<2.5 \mathrm{mmol} / \mathrm{L}$ & $15(9.3)$ & $15(27.8)$ & $0(0.0)$ \\
\hline $2.5-<5.0 \mathrm{mmol} / \mathrm{L}$ & $39(24.1)$ & $39(72.2)$ & $0(0.0)$ \\
\hline$\geq 5.0-10.0 \mathrm{mmol} / \mathrm{L}$ & $94(58.0)$ & $0(0.0)$ & 94 (87.0) \\
\hline$>10.0 \mathrm{mml} / \mathrm{L}$ & $14(8.6)$ & $0(0.0)$ & $14(13.0)$ \\
\hline Median (IQR) & $6.3(4.5-8.0)$ & $3.7(2.3-4.5)$ & $7.4(6.3-8.6)$ \\
\hline
\end{tabular}

$\mathrm{IQR}=$ interquartile ratio.

*Age-specific cut-offs for respiratory rate, pulse rate, body temperature, Blantyre coma score, and oxygen saturation as described in Table 2.

† Participants can have more than one emergency sign; total percentage may be more than 100. 
TABLE 2

Age-specific cut-offs for severely deranged vital signs

\begin{tabular}{llc}
\hline \multicolumn{1}{c}{ Vital sign } & \multicolumn{1}{c}{ Age } & Severely deranged \\
\hline Respiratory rate, breaths/min & $<1$ month & $<20$ or $>80$ \\
& 1 month- $<1$ year & $<15$ or $>60$ \\
& $1-<5$ years & $<10$ or $>50$ \\
& $5-12$ years & $<8$ or $>40$ \\
& $>12$ years & $<8$ or $>30$ \\
Saturation, \% & All & $<90$ \\
Pulse rate, beats/min & $<1$ month & $<80$ or $>200$ \\
& 1 month- $<1$ year & $<80$ or $>180$ \\
& $1-<5$ years & $<70$ or $>170$ \\
& $5-12$ years & $<60$ or $>150$ \\
Blantyre coma score & $>12$ years & $<40$ or $>130$ \\
Axillary temperature, ${ }^{\circ} \mathrm{C}$ & All & $\leq 2 / 5$ \\
& $<1$ month & $<35.5$ or $>38.5$ \\
& $\geq 1$ month & $<34$ or $>40$ \\
\hline
\end{tabular}

The cut-offs were derived from a previous study based on local and international guidelines for age specific cut-offs.

history of previous admissions and other illnesses was taken. The presence of any of the above-mentioned conditions or more than three admissions in the past 12 months was considered as a chronic illness.

Venous blood samples were collected for all enrolled participants immediately after enrolment, and the time of collection was documented. Cortisol samples were collected in a vacutainer blood collection tube containing spray-coated silica and a gel for serum separation. Blood was drawn using a syringe from the cannula or directly from the vein into the collecting tubes. The cortisol samples were centrifuged within 30 minutes of collection and then immediately stored in a freezer at $-10^{\circ} \mathrm{C}$ or less until transported in a cooler box for analysis at a private laboratory outside the College of Medicine and Queen Elizabeth Central Hospital. Cortisol samples were analyzed using chemiluminescent microparticle immunoassay technology using ARCHITECT Cortisol assay (Abbot Laboratory, Abbot Park, IL) ${ }^{22}$ to quantitatively determine the serum cortisol. A cortisol level of $<25 \mu \mathrm{g} / \mathrm{dL}$ ( $690 \mathrm{nmol} / \mathrm{L}$ ) was considered low, ${ }^{14,23}$ and a cortisol level $<10 \mu \mathrm{g} / \mathrm{dL}(276 \mathrm{nmol} /$ L) was considered adrenal insufficiency. ${ }^{15}$

To calculate the sample size, the proportion of low cortisol in the controls was estimated at $20 \%$ based on a prevalence from another study conducted in patients with severe illness. ${ }^{24}$ The proportion in severely sick children with low blood glucose was assumed to be $45 \%$. Using the Fleiss formula for correlation $^{25}$ with a power of $80 \%$ and a significance level of 0.05 , we calculated a required total sample of 150 children (50 cases and 100 controls).

Data were entered into Epi info version 7.4 (CDC, Atlanta, GA) and then exported to Microsoft Excel (Microsoft Corporation, Redmond, WA) for data cleaning. Stata version 14 (StataCorp, College Station, TX) was used for analysis. Basic characteristics of all the study participants were described using proportions, median and means as appropriate. Characteristics of the cases was compared with controls. A $\chi^{2}$ test was conducted to analyze if the proportion of children with low cortisol was different between cases and controls. Multivariable conditional logistic regression was conducted using the following variables selected by the investigators based on clinical plausibility: 1) sex, 2) Presence of severely deranged vital signs as defined in Table 2, 3) prolonged fasting ( $>8$ hours), 4) chronic illness as described in the Methods section, 5) time of blood sample collection categorized as morning (7:30 AM to 12:00 PM) and afternoon (after 12 PM to 4:30 PM), and 6) whether a participant was referred from another facility. The cut-offs for determining severely deranged vital signs (Table 2) were derived from a previous study based on local and international guidelines for age specific cut-offs. ${ }^{9}$ Conditional logistic regression analysis of the association of cortisol and blood glucose level was also conducted.

All eligible participants and their guardians were given oral and written information about the study from the study nurse. An informed consent form was read out loud to the study participants and their guardians, which included information on the reason for conducting the study and potential harm or discomfort caused by study procedures. For all children aged 7 years and above, an assent was obtained in addition to the consent from the guardian. Participants were free to withdraw their participation in the study or use of their data at any point.

An approval to conduct the study was obtained from College of Medicine Research Ethics Committee for ethical review P.02/19/2589. Consent to conduct the study was sought from the hospitals' directors and the head of the pediatric department at the College of Medicine.

\section{RESULTS}

Baseline characteristics of study participants. In this case-control study, a total of 162 children were enrolled (54 cases; 108 controls). Table 1 gives an overview of background characteristics of included children. The median age was 2.8 years (interquartile range [IQR]: 1.7-4.4). There were in total $61 / 162$ (37.6\%) female subjects, with 18/54 (33.3\%) female subjects among cases versus 43/108 (39.3\%) female subjects among controls. Most of the patients (122/162; $76.3 \%)$ presented with at least one severely deranged vital sign. More than half $(96 / 162 ; 59.3 \%)$ of the study participants were enrolled at Zomba hospital. Background characteristics were distributed equally between cases and controls except for fasting for more than 8 hours prior to study inclusion, which was more common among cases than controls (Table 1). The median cortisol level was $58.9 \mu \mathrm{g} / \mathrm{dL}(1,623$ $\mathrm{nmol} / \mathrm{L}$; IQR, 42.3-68.1 $\mu \mathrm{g} / \mathrm{dL})$ in cases and $40.9 \mu \mathrm{g} / \mathrm{dL}$ $(1,092 \mathrm{nmol} / \mathrm{L} ;$ IQR: 33.7-51.2 $\mu \mathrm{g} / \mathrm{dL})$ in controls $(P=0.834)$ (Table 3).

TABLE 3

Proportion of low cortisol among cases and controls

\begin{tabular}{cccc}
\hline Variable & All $(N=162)$ & Cases $(N=54)$ & Controls $(N=108)$ \\
\hline Cortisol levels, $n(\%)$ & & & \\
$<25 \mu \mathrm{g} / \mathrm{dL}$ & $13(8.0)$ & $4(7.4)$ & $9(8.3)$ \\
$>25 \mu \mathrm{g} / \mathrm{dL}$ & $149(92.0)$ & $50(92.6)$ & $99(91.6)$ \\
Median (IQR) & $43.5(35.1-60.8)$ & $58.9(42.3-68.1)$ & $40.9(33.7-51.2)$ \\
\hline
\end{tabular}

$\mathrm{IQR}=$ interquartile range

${ }^{*}$ Chi-square $P$ value. 
TABLE 4

The association of low cortisol with low blood glucose

\begin{tabular}{|c|c|c|c|c|c|c|}
\hline Variable & UOR & $P$ value & $95 \% \mathrm{Cl}$ & $\mathrm{AOR}^{*}$ & $P$ value & $95 \% \mathrm{Cl}$ \\
\hline Cortisol levels (Ref $>25$ ) & 0.6 & 0.604 & $0.1-3.4$ & 0.3 & 0.312 & $0.04-3.0$ \\
\hline Severely deranged vital sign & 0.7 & 0.518 & $0.3-2.0$ & 0.7 & 0.495 & $0.2-2.0$ \\
\hline Fasting time (Ref $<8$ hours) & 1.1 & 0.818 & $0.5-2.5$ & 1.2 & 0.709 & $0.5-2.8$ \\
\hline Sex (Ref male) & 0.9 & 0.928 & $0.4-2.4$ & 0.9 & 0.812 & $0.3-2.4$ \\
\hline Referred & 0.9 & 0.891 & $0.3-3.0$ & 0.9 & 0.946 & $0.3-3.2$ \\
\hline Time of the day (Ref morning) & 1.1 & 0.761 & $0.5-2.7$ & 1.2 & 0.672 & $0.5-3.3$ \\
\hline
\end{tabular}

$\mathrm{AOR}=$ adjusted odds ratio; $\mathrm{Cl}$ = confidence interval; $\mathrm{UOR}$ = unadjusted odds ratio.

${ }^{*}$ Adjusted odds ratio for all the covariates in the table.

Association of serum cortisol and low glycemia. A total of $13 / 162(8.0 \%)$ patients fulfilled the diagnosis of low cortisol with a cortisol level of $<25 \mu \mathrm{g} / \mathrm{dL}$ (690 nmol/L). The proportion of low cortisol was $4 / 54(7.4 \%)$ in cases and $9 / 108$ (8.3\%) in controls $(P=0.834)$. The proportion of low cortisol was highest among hypoglycemic patients $(<2.5 \mathrm{mmol} / \mathrm{L})$; the proportion was $3 / 15(20 \%)$ compared with $1 / 39(2.6 \%)$ in low glycemia (2.5-5.0 mmol/L) and 9/108 (8.33\%) in normal glycemia $(5.0-15.0 \mathrm{mmol} / \mathrm{L})$, but this was not significant $(P=0.105)$. None of the participants was diagnosed with adrenal insufficiency with serum cortisol of $<10 \mu \mathrm{g} /$ $\mathrm{dL}(276 \mathrm{nmol} / \mathrm{L})$.

Logistic regression showed no association between low blood glucose and low cortisol (adjusted odds ratio: 0.33; 95\% Cl: 0.04-3.02) (Table 4). Linear regression analysis did not show any significant change in blood glucose level with an increase in cortisol levels ( $\beta$ coefficient: $-0.004 ; 95 \% \mathrm{Cl}$ : -0.020 to 0.012 ).

\section{DISCUSSION}

In this study of severely sick children in Malawi, the proportion of low cortisol among those with low blood glucose concentrations was not different from those with normal blood glucose levels. No association was found between low serum cortisol and low blood glucose.

These findings are contrary to other studies that have shown an association between low cortisol levels and low blood glucose levels. ${ }^{26,27}$ Adrenal insufficiency, or low cortisol, occurs in severe illness, and some studies have reported prevalence as high as $60 \%$ among severely ill adult patients with septic shock. ${ }^{13}$ In this study we hypothesized that low glycemia could be a manifestation of low cortisol or adrenal insufficiency in severe illness.

Although adrenal insufficiency has reportedly been high among septic patients, studies reporting an association between low cortisol and low blood glucose levels were conducted in patients without severe illness. ${ }^{26,27}$ Hence, the lack of association in this study may be explained by a disruption of the normal metabolic balance where glucose variability occurs in severe illness, causing an unpredictable cortisol-glucose relationship. ${ }^{20,28,29}$

In the studied children, $8 \%$ fulfilled the criteria of low cortisol. However, the distribution was similar between cases and controls. These children possibly had a disruption of the hypothalamic-pituitary-adrenal axis related to the severe

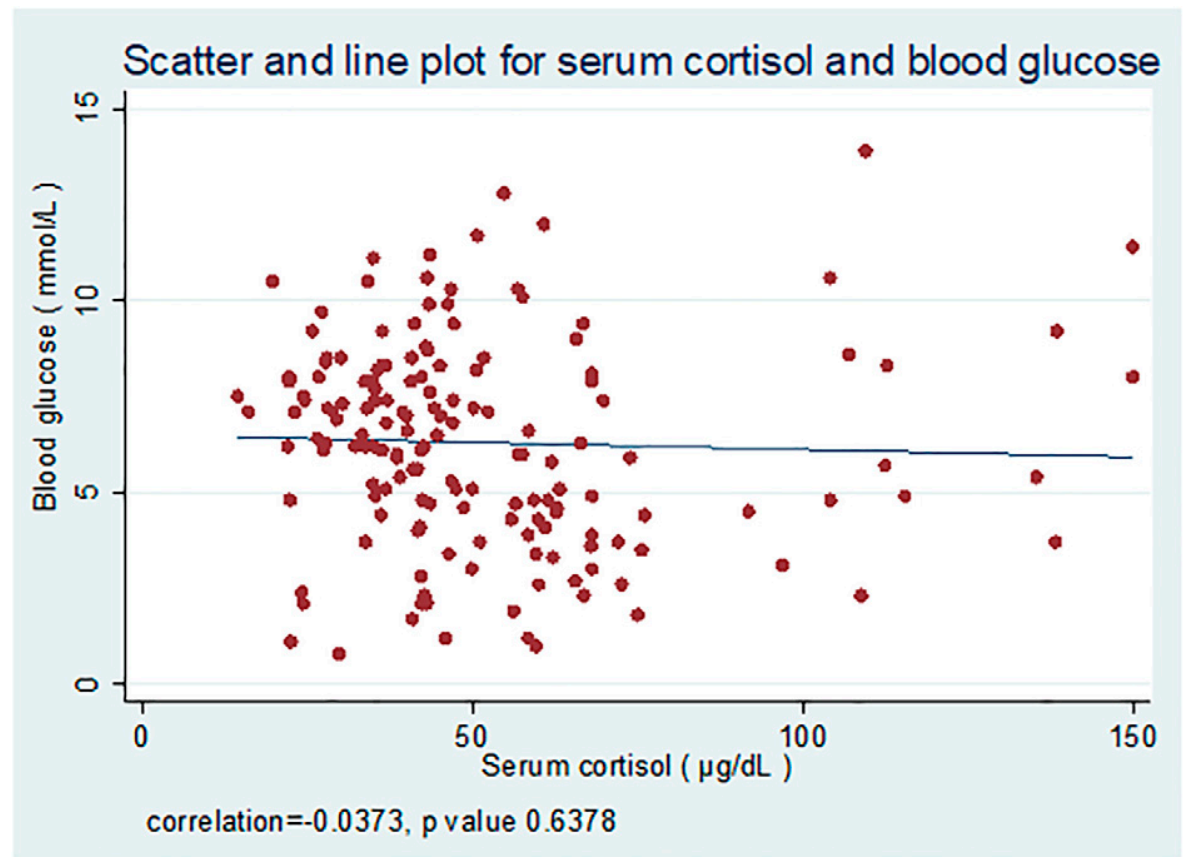

FIGURE 1. Line and scatter plot showing the relationship between cortisol levels and blood glucose levels. This figure appears in color at www. ajtmh.org. 
illness and were either not able to secrete as much cortisol in response to the stimulus triggered by severe disease or inactivated the cortisol faster. ${ }^{30}$ However, no child had an overt adrenal insufficiency with cortisol values of $<10 \mu \mathrm{g} / \mathrm{dL}$. It may be that children with illness-induced adrenal insufficiency would not survive the initial phase of severe acute illnesses and would die before arrival to hospital because high mortality has been seen in patients with adrenal insufficiency. ${ }^{31,32}$

Our results suggest that the mechanisms for low or hypoglycemia are not simply explained by falling cortisol values and that more complex interactions should be considered. The reason for low glycemia in severely sick children must be sought elsewhere. It could possibly be due to fasting and poor feeding in severe illness ${ }^{33}$ coupled with poor reserves of glucose due to underlying moderate malnutrition, ${ }^{34}$ which is common in the study setting. ${ }^{35}$ Severe illness increases catabolic demands for glucose, diminishing body reserves of glucose in combination with a limited intake of glucose. ${ }^{36}$ Indeed, the proportion of fasting in this study was much higher among cases than controls and suggests that fasting plays an important role in the development of low glycemia and hypoglycemia. We also noted a higher proportion of controls had severe respiratory distress compared with cases, which may suggest that low blood glucose levels are common in certain conditions.

Conversely, $26 \%$ children had a noticeable high cortisol level (>60 $\mu \mathrm{g} / \mathrm{dL})$, indicating high stress levels due to acute severe disease. The observed cortisol levels in our study were high, such that $26 \%$ children had cortisol level $>60$ $\mu \mathrm{g} / \mathrm{dL}$. However, the values were similar to other published studies conducted in children with septic shock admitted in the intensive care unit. ${ }^{37,38}$ A study conducted in critically ill children found that children who developed adrenal insufficiency had higher cortisol at baseline as compared with children who did not develop adrenal insufficency. ${ }^{39}$

There are a number of limitations to this study. Though study participants were severely ill, one low glucose reading might not indicate whether the low glycemia was persistent. Although there were too few cases to make statistical conclusions, there was a tendency for those with hypoglycemia (blood glucose $<2.5 \mathrm{mmol} / \mathrm{L}$ ) to be more likely to have low cortisol levels, and thus we may have reported different results with another definition of low glycemia than blood glucose of $<5 \mathrm{mmol} / \mathrm{L}$. In addition, the included children had a wide range of symptoms and diagnoses, making the study population heterogeneous, which may have affected our results. Matching for the presenting WHO emergency sign would have possibly produced different results because a higher proportion of certain emergency signs has been noted in controls. Most other studies that have been conducted on low cortisol were done in patients with septic shock in an intensive care unit. ${ }^{13,38}$ Possible overestimation of the proportion difference used in the sample size calculation may have led to an underpowered sample size. However, a proportion of $45 \%$ was chosen because a smaller proportion was considered of less clinical significance.

\section{CONCLUSION}

Though low cortisol was present in $8 \%$ of the study population, no association between low glycemia and low cortisol levels was seen in severely ill children admitted to tertiary referral hospitals in Malawi. The explanation for hypoglycemia among these children should be sought among alternative mechanisms.

Received January 11, 2021. Accepted for publication April 14, 2021. Published online July 19, 2021.

Acknowledgments: The authors thank all study participants for taking part in the study and Queen Elizabeth Central Hospital and Zomba Central Hospital management teams for allowing us to conduct the study in the hospitals. The authors are also grateful to the research study team Henderson Masanjala, Naomi Muheka, Helen Noniwa and Juliet Mphande for the dedicated work with the study.

Financial support: The study was funded by a project grant from Swedish Research Council (Vetenskapsrådet 348-2014-2791) and Ollie and Elof Ericssons Stiftelse. The funders had no role in the study design, data collection and analysis, interpretation of data, writing of the report, or submission for publication.

Authors' addresses: Fatsani Ngwalangwa and Josephine Langton, University of Malawi, College of Medicine, Blantyre, Malawi, E-mails: fatsa400@yahoo.com and jlangton@medcol.mw. Clifford Katumbi, Light House Trust, Kamuzu Central Hospital Area 33, Lilongwe, Malawi, E-mail: cliffmkatu@gmail.com. Queen Dube, Queen Elizabeth Central Hospital, Paediatrics Department, Blantyre, Malawi, E-mail: drdubefirst@yahoo.com. Tim Baker, Paediatric Department, University of Malawi, College of Medicine, Blantyre, Malawi, Department of Global Public Health, Health Systems and Policy Group, Karolinska Institutet, Sweden, and Department of Clinical Research, London School of Hygiene and Tropical Medicine, London, United Kingdom, E-mail: tim.baker@ki.se. Annika Janson, Astrid Lindgren Children's Hospital, Karolinska University Hospital, Stockholm, Sweden, and Karolinska Institutet, Department of Women's and Children's Health, Stockholm, Sweden, E-mail: annika.janson@sll.se. Helena Hildenwall, Astrid Lindgren Children's Hospital, Karolinska University Hospital, Sweden, and Department of Global Public Health, Health Systems and Policy Group, Karolinska Institutet, Sweden, E-mail: helena.hildewall@ki.se.

This is an open-access article distributed under the terms of the Creative Commons Attribution (CC-BY) License, which permits unrestricted use, distribution, and reproduction in any medium, provided the original author and source are credited.

\section{REFERENCES}

1. Mergenthaler P, Lindauer U, Dienel GA, Meisel A, 2013. Sugar for the brain: the role of glucose in physiological and pathological brain function. Trends Neurosci 36: 587-597.

2. Sprague JE, Arbeláez AM, 2011. Glucose counterregulatory responses to hypoglycemia. Pediatr Endocrinol Rev 9: 463-475.

3. Faustino EVS, Hirshberg EL, Bogue CW, 2012. Hypoglycemia in critically ill children. J Diabetes Sci Technol 6: 48-57.

4. WHO, 2013. Hospital Care for Children: Guidelines for the Management of Common Illnesses with Limited Resources. Geneva, Switzerland: World Health Organization.

5. Osier FHA, Berkley JA, Ross A, Sanderson F, Mohammed S, Newton CRJC, 2003. Abnormal blood glucose concentrations on admission to a rural Kenyan district hospital: prevalence and outcome. Arch Dis Child 88: 621-625.

6. Elusiyan JB, Adejuyigbe EA, Adeodu OO, 2006. Hypoglycaemia in a Nigerian paediatric emergency ward. $J$ Trop Pediatr 52: 96-102.

7. Willcox ML, Forster M, Dicko Ml, Graz B, Mayon-White R, Barennes $\mathrm{H}, 2010$. Blood glucose and prognosis in children with presumed severe malaria: is there a threshold for 'hypoglycaemia'? Trop Med Int Health 15: 232-240.

8. Nadjm B, Mtove G, Amos B, Hildenwall H, Najjuka A, Mtei F, Todd $\mathrm{J}$, Reyburn H, 2013. Blood glucose as a predictor of mortality in children admitted to the hospital with febrile illness in Tanzania. Am J Trop Med Hyg 89: 232-237. 
9. Ngwalangwa $\mathrm{F}$, Phiri $\mathrm{CHA}$, Dube $\mathrm{Q}$, Langton J, Hildenwall $\mathrm{H}$, Baker T, 2019. Risk factors for mortality in severely ill children admitted to a tertiary referral hospital in Malawi. Am J Trop Med Hyg 101: 670-675.

10. Baker T, Ngwalangwa F, Masanjala H, Dube Q, Langton J, Hildenwall $\mathrm{H}, 2020$. Mortality impact of an increased blood glucose cut-off level for hypoglycaemia treatment in severely sick children aged 1 month to 5 years in Malawi - the SugarFACT randomised controlled trial. Lancet Global Health 8: e1546-e1554.

11. Sibbald WJ, Short A, Cohen MP, Wilson RF, 1977. Variations in adrenocortical responsiveness during severe bacterial infections. Unrecognized adrenocortical insufficiency in severe bacterial infections. Ann Surg 186: 29-33.

12. Span LFR, Hermus ARMM, Bartelink AKM, Hoitsma AJ, Gimbrère JSF, Smals AGH, Kloppenborg PWC, 1992. Adrenocortical function: an indicator of severity of disease and survival in chronic critically ill patients. Intensive Care Med 18: 93-96.

13. Annane D, Maxime V, Ibrahim F, Alvarez JC, Abe E, Boudou P, 2006. Diagnosis of adrenal insufficiency in severe sepsis and septic shock. Am J Respir Crit Care Med 174: 1319-1326.

14. Zaloga GP, Marik P, 2001. Hypothalamic-pituitary-adrenal insufficiency. Crit Care Clin 17: 25-41.

15. Marik PE, Pastores SM, Annane D, Meduri GU, Sprung CL, Arlt W, etal., 2008. Recommendations for the diagnosis and management of corticosteroid insufficiency in critically ill adult patients: consensus statements from an international task force by the American College of Critical Care Medicine. Crit Care Med 36: 1937-1949.

16. WHO, 2016. Guideline: Updates on Paediatric Emergency Triage, Assessment and Treatment: Care of Critically-III Children. Geneva, Switzerland: World Health Organization. Available at: https://www.ncbi.nlm.nih.gov/books/NBK350528/.

17. Okiro EA, Kazembe LN, Kabaria CW, Ligomeka J, Noor AM, Ali D, Snow RW, 2013. Childhood malaria admission rates to four hospitals in Malawi between 2000 and 2010. PLoS One 8: e62214.

18. Robison J, Ahmed Z, Durand C, Nosek C, Namathanga A, Milazi R, Thomas A, Mwansambo C, Kazembe P, Torrey S, 2011. Implementation of ETAT (Emergency Triage Assessment And Treatment) in a central hospital in Malawi. Arch Dis Child (Suppl 1): A74-A75.

19. Patki VK, Chougule SB, 2014. Hyperglycemia in critically ill children. Indian J Crit Care Med 18: 8-13.

20. Pérez-Calatayud ÁA, Guillén-Vidaña A, Fraire-Félix IS, AnicaMalagón ED, Briones Garduño JC, Carrillo-Esper R, 2017. Metabolic control in the critically ill patient an update: hyperglycemia, glucose variability hypoglycemia and relative hypoglycemia. Cir Cir 85: 93-100.

21. Newton CR, Chokwe T, Schellenberg JA, Winstanley PA, Forster D, Peshu N, Kirkham FJ, Marsh K, 1997. Coma scales for children with severe falciparum malaria. Trans $R$ Soc Trop Med Hyg 91: 161-165.

22. Konforte D, Bozovic A, Martens P, Yip P, 2011. Validation of the cortisol method on the abbott architect (R) i2000 immunoassay system for measurement of urine cortisol by comparison to LCMS-MS. Clin Biochem 44: 1175-1175.
23. Sternberg EM, Chrousos GP, Wilder RL, Gold PW, 1992. The stress response and the regulation of inflammatory disease. Ann Intern Med 117: 854-866.

24. Beishuizen A, Thijs LG, 2001. Relative adrenal failure in intensive care: an identifiable problem requiring treatment? Best Pract Res Clin Endocrinol Metab 15: 513-531.

25. Fleiss, J, 1981. Statistical Methods for Rates and Proportions, 2nd edition. New York, NY: Wiley.

26. Arlt W, 2008. Adrenal insufficiency. Clin Med (Lond) 8: 211-215.

27. Artavia-Loria E, Chaussain JL, Bougnères PF, Job JC, 1986. Frequency of hypoglycemia in children with adrenal insufficiency. Acta Endocrinol (Copenh) 113: S275.

28. Waeschle RM, Moerer O, Hilgers R, Herrmann P, Neumann P, Quintel M, 2008. The impact of the severity of sepsis on the risk of hypoglycaemia and glycaemic variability. Crit Care 12: R129.

29. Wintergerst KA, Buckingham B, Gandrud L, Wong BJ, Kache S, Wilson DM, 2006. Association of hypoglycemia, hyperglycemia, and glucose variability with morbidity and death in the pediatric intensive care unit. Pediatrics 118: 173-179.

30. Levy-Shraga Y, Pinhas-Hamiel O, 2013. Critical illness-related corticosteroid insufficiency in children. Horm Res Paediatr 80: 309-317.

31. Shulman DI, Palmert MR, Kemp SF, 2007. Adrenal insufficiency: still a cause of morbidity and death in childhood. Pediatrics 119: e484-e494.

32. Menon K, Ward RE, Lawson ML, Gaboury I, Hutchison JS, Hébert PC, Canadian Critical Care Trials G, 2010. A prospective multicenter study of adrenal function in critically ill children. Am J Respir Crit Care Med 182: 246-251.

33. Ngwalangwa F, Chirambo CM, Lindsjö C, Dube Q, Langton J, Baker T, Hildenwall H, 2020. Feeding practices and association of fasting and low or hypo glycaemia in severe paediatric illnesses in Malawi - a mixed method study. BMC Pediatr 20: 423.

34. Emery PW, 2005. Metabolic changes in malnutrition. Eye (Lond) 19: 1029-1034.

35. National Statistical Office (NSO) [Malawi] and ICF, 2017. Malawi Demographic and Health Survey 2015-16. Zomba, Malawi and Rockville, MD: NSO and ICF.

36. Zijlmans W, van Kempen A, Ackermans M, de Metz J, Kager P, Sauerwein H, 2008. Glucose kinetics during fasting in young children with severe and non-severe malaria in Suriname. Am $J$ Trop Med Hyg 79: 605-612.

37. Lin M, Zhang B-Q, Lin H, Sun P-M, Wang S-B, Guo R-M, Weng B, Guo X-F, 2020. Dynamic changes in serum cortisol and Acth levels and lymphocyte subset counts in children with septic shock: Research Square. doi: 10.21203/rs.3.rs-39009/v1.

38. Rady HI, Aly YS, Hafez M, Bazaraa HM, 2014. Adrenocortical status in infants and children with sepsis and septic shock. Gaz Egypt Paediatr Assoc 62: 18-23.

39. Karagüzel G, Atay $S$, Değer $O$, Imamoğlu M, Ökten A, Karagüzel G, 2012. The effects of three specific conditions related to critical care on adrenal function in children. Intensive Care Med 38: 1689-1696. 